\title{
Joint Optimization of Energy Consumption and Throughput of Directional WMNs
}

\author{
Iyad Lahsen Cherif ${ }^{1}$, Lynda Zitoune ${ }^{2,1}$, Véronique Vèque ${ }^{1}$ \\ ${ }^{1}$ Signals and Systems Laboratory, (L2S, UMR CNRS 8506) \\ CentraleSupelec - CNRS - University Paris-Sud - University Paris-Saclay \\ 3, Rue Joliot-Curie, 91192 Gif sur Yvette, France. \\ \{iyad.lahsen-cherif, Veronique.VEQUE\} @l2s. centralesupelec.fr \\ ${ }^{2}$ Department of Systems Engineering, ESIEE-Paris \\ 2, boulevard Blaise Pascal, 93162 Noisy le Grand, France. \\ lynda.zitounedesiee.fr
}

\begin{abstract}
Directional Antennas (DAs) provide higher gain, and reduce interference by directing beams toward the desired receiver. In this paper, we propose a new joint optimization framework considering the energy consumption and throughput in DAs Wireless Mesh Networks (WMNs). We formulate the joint optimization problem as a Mixed Integer Linear Problem (MILP) using a weighted objective function of both the consumed energy and the throughput. We use ILOG Cplex [1], a software based on branch and cut method, to find the optimal solution of the optimization problem. Results prove the efficiency of using DAs in WMNs in the considered scenarios and show that the consumed energy increases with the beamwidth and decreases with the number of power levels when using power control.
\end{abstract}

\section{INTRODUCTION}

Many blocking factors such as poor communication infrastructure, or lack of energy suppliers are a major barrier of Internet penetration in isolated rural and sub-urban areas. The lack of infrastructure requires higher costs to the operators for network deployment, planning and maintenance, wired lines to connect BSs, default electrical grids, or generators. An alternative approach to deal with this issue is the use of a self-configured Wireless Mesh Network (WMN) [2] to connect outdoor multimode femtocells to a remote base station as developed in the $\mathrm{LCI}_{4} \mathrm{D}^{1}$ project. A multimode femtocell is a low power node (LPN) embedding both cellular (4G/LTE) and mesh WiFi technologies. The mesh nodes form a local backhaul network to gather and relay data from cellular devices to the macro base station through an edge gateway. However, the shortage of energy in these settlements leads to optimize the energy consumption of the wireless backhaul network.

Directional Antennas (DAs) proved their efficiency in terms of improving the network throughput and reducing the consumed energy [3]. DAs have the ability to focus the transmission toward the desired receiver, without radiating in all direction as Omni-directional Antennas (OAs). DAs technology has many advantages for WMNs where the communication between two nodes are multi-hop. Indeed farthest nodes can be reached thanks to the high antenna gain of DAs, improving the transmission range. Furthermore, spatial reuse is improved

\footnotetext{
${ }^{1}$ Low Cost Infrastructure for Developing Countries
}

and concurrent transmissions can occur without collisions. As demonstrated in our previous work [3], more the antennas are directive, less is the number of links in a communication path. Hence, packets are forwarded through less intermediate hops to the destination. Furthermore, using intensive simulation experiments we show also that DAs improve the network throughput and reduce the consumed energy.

In this paper, we formulate an optimization problem that jointly optimizes the throughput and the energy consumption. To the best of our knowledge, no previous optimization models of DAs networks considered jointly the energy consumption and the throughput. Previous work were limited to OAs networks [4]-[6] and throughput optimization in DAs networks [7].

This paper is organized as follows. In the next section we present related work. The model is presented in Section III. In Section IV, we formulate the joint energy consumption and throughput optimization problem. We outline the objective function and the constraints. Simulation results are presented in Section V. Finally, we conclude the paper in Section VI.

\section{RELATED WORK}

Several works have been proposed to optimize the network performance in terms of throughput and energy consumption considering either OAs or DAs.

In the context of OAs network, a Mixed Integer Linear Program (MILP) is proposed in [4] to jointly optimize the energy and throughput. It proposes an heuristic based on AntColony to reduce the complexity of the solution and shows that its can save up to $20 \%$ in energy consumption. Joint design and management optimization are considered in [8], [9] in order to reduce the deployment cost and the consumed energy and show a gain of almost $30 \%$ of energy saving. Energy and throughput are jointly optimized in [5] using continuous power control and multi-rate transmissions. An Integer Linear Programing (ILP) problem \& Iterative Local Search [7] in a multi-channel network is used to optimize jointly the consumed energy and the throughput. Packet loss ratio and throughput are optimized in [10] using Topology control and the Routing Assignment (TORA). A Mixed Integer Linear Programing (MILP) approach for energy consumption 
and the end-to-end delay optimization is proposed in [6], providing $16 \%$ gain.

A few works consider DAs. An ILP problem for a multichannel network is formulated in [11]. It shows an enhancement of $14 \%$ in PDR (Packet Delivery Ratio) by increasing the number of available channels from 4 to 6 . The Iterative Local Search is used to optimize jointly the end-to-end delay and the throughput. A Max-Min flow problem is used to model minimization of the number of DAs in order to improve network reliability [12]. For a $100 \%$ reliability, the increase in the number of antennas is of $80 \%$ on average. A joint link scheduling and power control optimization problem is considered in [13] to improve the network throughput. Multichannel DAs network is considered in [14] where the packet Delivery ratio, the average delay and the throughput are improved by up $57 \%$. However, all these works were limited on throughput optimization and do not consider the energy consumption in the objective function.

To the best of our knowledge, no previous work tackled the problem of joint energy consumption and throughput in DAs using an optimization approach. In this paper, we address the problem of joint energy consumption minimization and throughput maximization in DAs networks.

\section{SYSTEM MODEL}

\section{A. Interference Model}

Various interference models have been used in OAs considering either the locations of nodes or the received power as a metric to characterize the interference. However, these models are not valid in DAs networks, since (i) some directions are free of interference because DAs radiate only toward a given direction and (ii) DAs can reach farther nodes since the directional range is extended.

In this work we consider a new interference model by combining the ones used in [15] to compute the overlap count representing the number of overlaps between beams, and in [13], [16] to insure a minimum distance between nodes. It takes into account the position and distance between nodes.

Let $(i, j)$ and $(p, q)$ two pairs of transmitting-receiving nodes, at positions $\left(X_{i}, X_{j}\right)$ and $\left(X_{p}, X_{q}\right)$, respectively. Communications between pairs $(i, j)$ and $(p, q)$ can occur simultaneously, without generating interference, if and only if:

$$
\begin{gathered}
\left|X_{i}-X_{j}\right| \geq(1+\nu)\left|X_{k}-X_{j}\right| \\
\left(X_{i} \widehat{X_{j}, X_{i}} X_{p}\right) \geq \gamma \quad \text { and } \quad\left(X_{p} \widehat{X_{q}, X_{p}} X_{i}\right) \geq \gamma
\end{gathered}
$$

where $|$.$| is the euclidean distance, \gamma$ is a threshold angle and $\nu$ represents a guard zone to prevent a neighboring node from transmitting at the same time.

Hence, the set of interferers to the communication between nodes $i$ and $j$ is defined as follows:

$\mathbb{I}_{(i, j)}^{(p, q)}=\left\{(p, q) \in \mathcal{I}^{2}\right.$ s.t. Eq. (1) and Eq. (2) are not verified $\}$

\section{B. Energy Model}

We consider that each node have three states, either (i) transmitting, (ii) receiving, or (iii) idle. A node is in an active state, transmitting or receiving if it has some packets to send, forward or receive, respectively. Otherwise, it goes to the idle state.

\section{Power Control model}

We consider that each node has many transmission levels, $\mathcal{L}=\{1, \ldots,|\mathcal{L}|\}$, available to use depending on the location of the next hop. In this way, energy can be saved.

Let consider that a node has three power levels, either transmitting at full power $P_{\max }$, at $\frac{P_{\max }}{2}$, or not transmitting 0 , and suppose that the next hop is close and can be reached using $\frac{P_{\max }}{2}$, therefore $\frac{P_{\max }}{2}$ of power is saved.

\section{PROBLEM FORMULATION}

In this section we define the optimization problem. Firstly, we start by presenting the variables and parameters, then we introduce the objective function followed by the problem constraints.

\section{A. Variables and Parameters}

In the following, we present the sets and parameters used in the optimization problem.

Sets:

- $\mathcal{I}=\{1, \ldots,|\mathcal{I}|\}$ : the set of nodes.

- $\mathcal{G}=\left\{g_{1}, \ldots, g_{n}\right\}$ the set of mesh gateways in the network.

- $\mathcal{U}=\{1, \ldots,|\mathcal{U}|\}$ the set of users generating the traffic.

- $\mathcal{L}=\{1, \ldots,|\mathcal{L}|\}$ the set of possible power levels that can be used by a node.

Parameters \& Input:

- Let $c_{i j}$ be the capacity of the link between nodes $i$ and $j$.

- Each user has an amount of traffic to send at time $t, d_{i, t}$.

- Each user is connected to its nearest Access Point (AP).

Binary variables:

- $x_{u, i j, t}^{l, L}$ is used to express the linking between two nodes and the beams used in communication at time $\mathrm{t}$ :

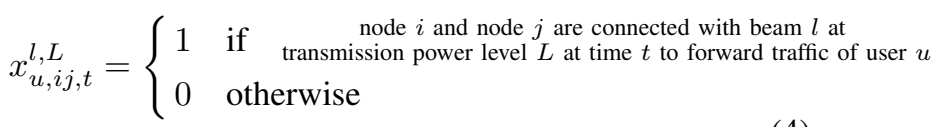

- The binary variable $a_{i, t}^{l, L}$ describes if node $i$ is transmitting at time $t$ using beam $l$ at power level $L . \forall i \in \mathcal{I}, \quad t \in$ $[0, T], \quad l \in \mathcal{B}_{i}, \quad L \in \mathcal{L}$

$$
a_{i, t}^{l, L}= \begin{cases}0 & \text { if } \sum_{j=1}^{|\mathcal{I}|} \sum_{u=1}^{|\mathcal{U}|} \sum_{l=1}^{\left|\mathcal{B}_{i}\right|} x_{u, i j, t}^{l, L}=0 \\ 1 \quad \text { otherwise } & \end{cases}
$$


- $b_{i, t}$ indicates if node $\mathrm{i}$ is receiving at time $t . \forall i \in \mathcal{I}, \quad t \in$ $[0, T]$

$$
b_{i, t}=\left\{\begin{array}{l}
0 \quad \text { if } \sum_{\substack{u=1 \\
\text { otherwise }}}^{|\mathcal{U}|} \sum_{k=1}^{|\mathcal{I}|} \sum_{l=1}^{\left|\mathcal{B}_{i}\right|} \sum_{L=1}^{|\mathcal{L}|} x_{u, k i, t}^{l, L}=0 \\
1 \quad \text { othis }
\end{array}\right.
$$

- $c_{i, t}$ indicates that node $i$ is on the idle state at time $t$.

$$
c_{i, t}= \begin{cases}1 & \text { if node } i \text { is at idle state at time } \mathrm{t} \\ 0 & \text { otherwise }\end{cases}
$$

- $u_{l i, t}$ is for user association.

$$
u_{l i, t}= \begin{cases}1 & \text { if } \text { user } l \text { is attached to node } i \text { at time slot } \mathrm{t} \\ 0 & \text { otherwise }\end{cases}
$$

Note that, to reduce the complexity of the neighbor discovery process and the optimization problem, we consider that reception is omnidirectional.

\section{B. Objective function}

The parameterised objective function is a weighted function which models the impact of DAs on the consumed energy and the throughput.

A node can be in three possible states: either (i) transmitting, (ii) receiving or, (iii) idle. Therefore, the total energy consumed by this node depends on its state at instant $t$.

The energy consumed by node $i$ at time $t$ is:

$$
\begin{aligned}
& \mathcal{E}_{i, t}=\underbrace{\sum_{l=1}^{\left|\mathcal{B}_{i}\right|} \sum_{L=1}^{|\mathcal{L}|} a_{i, t}^{l, L} \mathcal{E}_{\mathrm{TX}, L}}_{\begin{array}{c}
\text { Energy consumed when node } i \\
\text { is transmitting at powre level } \mathrm{L}
\end{array}}+\underbrace{b_{i, t} \mathcal{E}_{\mathrm{RX}}}_{\begin{array}{c}
\text { Energy consumed } \\
\text { if node } i \text { is receiving }
\end{array}} \\
& +\underbrace{\left(1-\sum_{l=1}^{\left|\mathcal{B}_{i}\right|} \sum_{L=1}^{|\mathcal{L}|} a_{i, t}^{l, L}\right) \cdot\left(1-b_{i, t}\right) \cdot c_{i, t} \mathcal{E}_{\text {idle }}}_{\begin{array}{c}
\text { Energy consumed } \\
\text { if node } i \text { is idle }
\end{array}}
\end{aligned}
$$

where $\mathcal{E}_{\mathrm{TX}, L}$ is the energy consumed by node $i$ if it is transmitting in the directional mode (use one beam) at level $L, \mathcal{E}_{\mathrm{RX}}$ if it is receiving and $\mathcal{E}_{\text {idle }}$ if it is idle.

The total energy consumed in the network is:

$$
\mathcal{E}=\sum_{t=1}^{T} \sum_{i=1}^{|\mathcal{I}|} \mathcal{E}_{i, t}
$$

The throughput can be modelled as:

$$
\mathcal{I} \mathcal{R}=\sum_{t=1}^{T} \sum_{i=1}^{|\mathcal{I}|} \sum_{L=1}^{|\mathcal{L}|} a_{i, t}^{l, L}
$$

where $\mathcal{I} \mathcal{R}$ stands for Inverse Rate.

Minimizing the time when APs are transmitting and receiving is equivalent to maximizing the rate.
Eq. (12) represents the objective function to minimize. It consists in minimizing the energy consumed by the network during the considered period and the network throughput.

$$
\min \frac{\alpha \mathcal{E}+(1-\alpha) \mathcal{I R}}{T}
$$

where $\alpha \in\{0,1\}$ is a weighted coefficient used to balance the energy consumed and the rate. If $\alpha=0$ then we mainly maximize the achievable rate and if $\alpha=1$ we minimize the energy consumed.

Next, we outline constraints of the joint energy consumption and throughput optimization problem.

\section{Constraints}

- Link capacity constraint:

$\forall(i, j) \in \mathcal{I} \times \mathcal{I}, \forall u \in \mathcal{U}, L \in \mathcal{L}, t \in[0, T], m \in \mathcal{B}_{i}$

$$
\sum_{u=1}^{|\mathcal{U}|} x_{u, i j, t}^{m, L} \times d_{u} \leq c_{i, j}
$$

Constraint (13) imposes that the traffic on links between nodes $i$ and $j$ do not exceed the total link capacity.

- Flow conservation (at node $i$ ):

$$
\begin{array}{r}
\sum_{u=1}^{|\mathcal{U}|} \sum_{t=1}^{T} \sum_{l=1}^{|\mathcal{L}|} \sum_{j=1}^{|\mathcal{I}|} \sum_{h=1}^{\left|\mathcal{B}_{i}\right|} x_{u, i j, t}^{h, L}=\sum_{u=1}^{|\mathcal{U}|} \sum_{t=1}^{T} \sum_{l=1}^{|\mathcal{L}|} \sum_{j=1}^{|\mathcal{I}|} \sum_{m=1}^{\left|\mathcal{B}_{j}\right|} x_{u, j i, t}^{m, L} \\
+\sum_{u=1}^{|\mathcal{U}|} w_{u i} \sum_{t=1}^{T} \sum_{l=1}^{|\mathcal{L}|} \sum_{j=1}^{|\mathcal{I}|} \sum_{m=1}^{\left|\mathcal{B}_{i}\right|} x_{u, i j, t}^{m, L} \quad \forall i \in \mathcal{I} \backslash\{G\}
\end{array}
$$

Constraint (14) represents that all the flows entering an intermediate node are forwarded to another. The left hand side corresponds to the number of flows going from node $i$, while the right hand side is the number of both flows coming from neighbors of node $i$ and flows generated by the attached users.

- Flow conservation at the gateway:

$$
\sum_{t=1}^{T} \sum_{l=1}^{|\mathcal{L}|} \sum_{i=1}^{|\mathcal{I}|} \sum_{m=1}^{\left|\mathcal{B}_{i}\right|} x_{u, i j, t}^{m, L}=1 \quad \forall u \in \mathcal{U} \text { and } j=|\mathcal{I}|+1
$$

Constraint (15) ensures that all the flows must arrive to the gateway $(|\mathcal{I}|+1)$.

- Interference constraint:

$$
x_{u, i j, t}^{m, L}+x_{u^{\prime}, p q, t}^{l, L^{\prime}} \mathbb{I}_{(i, j)}^{(p, q)} \leq 1
$$

$\forall(i, j),(p, q) \in \mathcal{I}^{2},\left(L, L^{\prime}\right) \in \mathcal{L}^{2}$, $t \in[0, T] ;\left(u, u^{\prime}\right) \in \mathcal{U}^{2},(m, l) \in \mathcal{B}_{i} \times \mathcal{B}_{p}$, where $\mathbb{I}_{(i, j)}^{(p, q)}$ is the set of interferers defined in Eq.(3). 
- One direction constraint:

$\sum_{l=1}^{\left|\mathcal{B}_{i}\right|} x_{u, i j, t}^{l, L} \leq 1 \quad \forall(i, j) \in \mathcal{I}^{2}, \forall u \in \mathcal{U}, L \in \mathcal{L}, t \in[1, T]$

- One power level constraint:

$$
\sum_{L=1}^{|\mathcal{L}|} x_{u, i j, t}^{l, L} \leq 1 \quad \forall(i, j) \in \mathcal{I}^{2}, \forall u \in \mathcal{U}, l \in \mathcal{B}_{i}, t \in[1, T]
$$

Constraints (18) and (17) are stated to ensure that an AP can transmit only at one power level, and in one direction in a given time.

- Simultaneous transmissions and receptions constraints:

$$
\sum_{u=1}^{|\mathcal{U}|} \sum_{l=1}^{|\mathcal{L}|} \sum_{j=1}^{|\mathcal{I}|} \sum_{m=1}^{\left|\mathcal{B}_{i}\right|} x_{u, i j, t}^{m, L}+\sum_{u=1}^{|\mathcal{U}|} \sum_{l=1}^{|\mathcal{L}|} \sum_{j=1}^{|\mathcal{I}|} \sum_{m=1}^{\left|\mathcal{B}_{i}\right|} x_{u, j i, t}^{m, L} \leq 1
$$

$\forall i \in \mathcal{I}$ and $t \in[0, T]$.

Constraint (19) prevents that a node transmits and receives at the same time slot.

- Loops avoidance

$\sum_{t=1}^{T} \sum_{l=1}^{|\mathcal{L}|} \sum_{j=1}^{|\mathcal{I}|} \sum_{m=1}^{\left|\mathcal{B}_{i}\right|} x_{u, i j, t}^{m, L} \leq 1$, and $\sum_{t=1}^{T} \sum_{l=1}^{|\mathcal{L}|} \sum_{j=1}^{|\mathcal{I}|} \sum_{m=1}^{\left|\mathcal{B}_{j}\right|} \sum_{n=1}^{\left|\mathcal{B}_{i}\right|} x_{u, j i, t}^{m, L}$

$\forall i \in \mathcal{I}, \forall u \in \mathcal{U}$.

Constraint (20) imposes that the traffic of a given user passes through a node only one time in order to prevent loops.

$\bullet$

$$
x_{u, i j, t}^{m, L}=0
$$

$\forall j \in \mathcal{I}$ and $t \in[0, T], i=|\mathcal{I}|+1, \forall L \in \mathcal{L}, \forall m \in$ $\mathcal{B}_{N+1}$.

Constraint (21) ensures that the traffic is not forwarded back to nodes after reaching the gateway.

- Binary variables constraints:

$$
\begin{gathered}
x_{u, i j, t}^{m, L}, \quad a_{i, t}^{m, L}, \quad b_{i, t}, \quad c_{i, t}, \quad u_{l i, t} \in\{0,1\}, \\
\forall(i, j) \in \mathcal{I}^{2}, \quad m \in \mathcal{B}_{i}, \quad L \in \mathcal{L}, \quad u \in \mathcal{U} .
\end{gathered}
$$

Equations (22) is to insure that variables take binary values. Equations (13 - 22) represent the optimization problem constraints.

In the next section, we present the evaluation methodology followed by the simulation results.

\section{Performance Evaluation}

\section{A. Evaluation methodology}

We use the ILOG Cplex ${ }^{2}$ solver [1] to find the optimal solution of the joint optimization problem presented in Eq. (12). To solve linear optimization problems, many software solutions were presented and compared in [17]. ILOG Cplex is the most efficient one in terms of resolution time and handled

\footnotetext{
${ }^{2}$ Java is used to implement the optimization model into Cplex solver
}

number of variables and it is based on the branch-and-cut algorithm [1].

- Inputs: The inputs are the simulation parameters summarized in Table I.

- Variables: Since the number of variables increases exponentially with the network size, we consider relatively small size networks equipped with a few number of beams for our simulations.

- Outputs: The outputs of the optimization problem are the set of variables $\left(x_{u, i j, t}^{l, L}\right)$ indicating which beam $l$, and power level $L$ are active in a given time slot $t$ for node $i$ to communicate with node $j$.

\section{B. Simulation Parameters}

In simulation experiments, we consider a WMN with a regular topology. The distribution of nodes follows a grid topology of 25 nodes equipped with DAs capabilities. We consider that nodes are deployed within an area of $1 \mathrm{~km} \times 1 \mathrm{~km}$ and spaced by $200 \mathrm{~m}$ in the $\mathrm{x}$ - and $\mathrm{y}$-axises. Only one gateway placed at the top-right corner of the grid is considered. We fix the transmission rate to $300 \mathrm{Kbps}$ for each node. Note that each node can either transmit or receive at a given time. We vary the beamwidth from $\frac{\pi}{6}$ to $2 \pi$.

$\leq$ The input values of the joint optimization problem are summarized in table I.

\begin{tabular}{|c|c||c|c|}
\hline Parameters & Values & Parameters & Values \\
\hline $\mathcal{E}_{\mathrm{Tx}}$ & 1 (Watt) & $|\mathcal{U}|$ & 50 \\
\hline $\mathcal{E}_{\mathrm{RX}}$ & 0.5 (Watt) & $|\mathcal{G}|$ & 1 (Top-right) \\
\hline $\mathcal{E}_{\text {idle }}$ & 0.1 (Watt) & Topology & Grid \\
\hline $\mathrm{L}$ & 1,2 , and 3 & $|\mathcal{I}|$ & $25(5 \times 5)$ \\
\hline$\alpha$ & {$[0,1]$} & $|\mathcal{B}|$ & {$[1,12]$} \\
\hline$\nu$ & 0.1 & $\gamma$ & $\frac{\pi}{3}$ \\
\hline
\end{tabular}

TABLE I: Simulations parameters

\section{Simulation Results}

We show the impact of the optimization weight $\alpha$, the beamwidth and the number of power levels on the consumed energy, the throughput and the consumed energy-throughput tradeoff.

For a given user traffic $u=1$, having node 1 as a source and node 25 as a destination (gateway), a $\frac{\pi}{4}$-beam DAs and 3 power levels, the output of the optimization problem is: $x_{1,1,2,1}^{1,1}=x_{1,2,8,2}^{2,1}=x_{1,8,15,2}^{2,3}=x_{1,15,25,4}^{3,2}=1$, where $x_{1,1,2,1}^{1,1}$ means that the traffic of user 1 is forwarded from node 1 to node 2 at time period 1 using beam number 2 and power level 1 , and otherwise 0 . For a user traffic $u=3$, having node 12 as a source: $x_{3,12,15,2}^{1,3}=x_{3,15,25,3}^{3,2}=1$, and otherwise 0 .

a) Impact of beamwidth: Remember that varying the value of the weight factor $\alpha$ leads to different optimization solutions. We consider the two extreme cases: (i) $\alpha=1$, we focus only on optimizing the energy consumption, (ii) $\alpha=0$, we maximize only the throughput, and we consider (iii) two intermediate cases, $\alpha=0.5$ and $\alpha=0.7$.

Figure 1 illustrates the energy consumed depending on the beamwidth for different values of the weight factor $\alpha$. We 
vary the beamwidth from $\frac{\pi}{6}$ to $2 \pi$. The energy consumption increases when the beamwidth gets larger. The maximum energy consumed is when the beamwidth is $2 \pi$ corresponding to an OAs network.

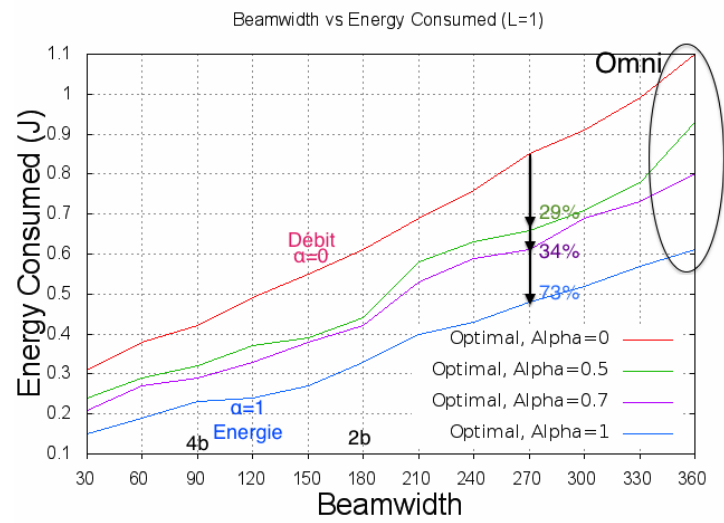

Fig. 1: Energy consumed vs BeamWidth for different values of $\alpha$ and for $\mathrm{L}=1$

Figure 2 shows that for the optimal solution, the throughput is decreasing in function of the beamwidth. Furthermore, for a given beamwidth, the closest $\alpha$ is to 1 , the worse is the throughput, and inversely, the closest $\alpha$ to 0 is, the better it is.

The throughput and energy consumed gains are summarized in Table II.

\begin{tabular}{|l|c|c|}
\hline$\alpha$ & Throughput & Consumed Energy \\
\hline \hline 0 & $34 \%$ & $0 \%$ \\
\hline 0.7 & $19 \%$ & $29 \%$ \\
\hline 0.5 & $12 \%$ & $34 \%$ \\
\hline 1 & $0 \%$ & $93 \%$ \\
\hline
\end{tabular}

TABLE II: Throughput and Energy consumption gains

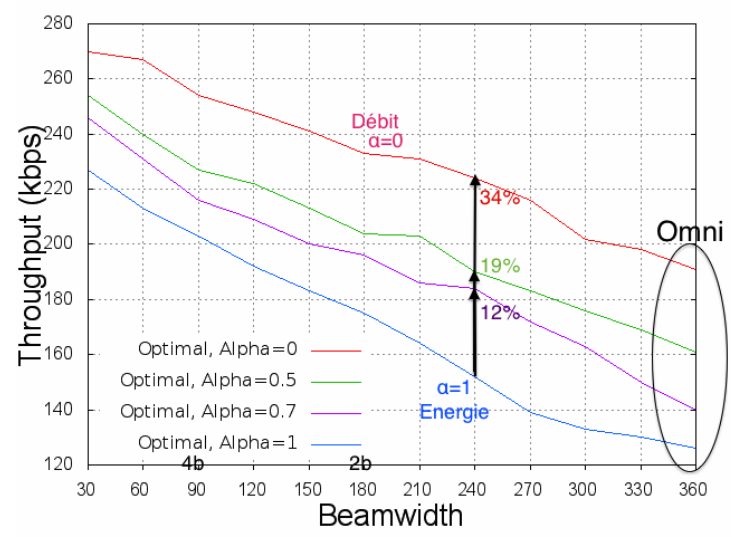

Fig. 2: Throughput vs Beamwidth for different values of $\alpha$ and for $\mathrm{L}=1$

b) Impact of Power Level: For simulations, we take the case of $|\mathcal{L}|=3$, that is, each node selects its transmit power from the set $\left\{0, \frac{P_{\max }}{2}, P_{\max }\right\}$. The impact of power control is considered in figure 3, where we show the energy consumed vs the beamwidth for different values of $L$, the number of power levels. The more power level are, the less energy is consumed. In fact, having more power levels allows to save more power. If the next hop can be reached with less power than the full power only, the node uses less power to reach the destination.

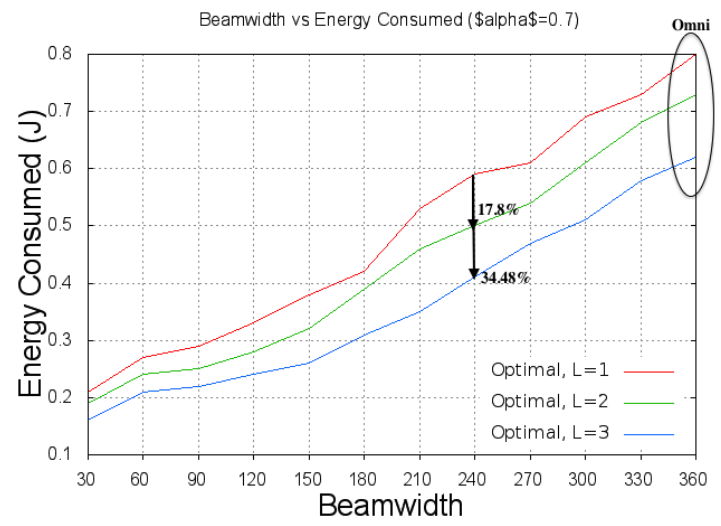

Fig. 3: Consumed Energy vs Beamwidth for different values of $L$ and for $\alpha=0.7$

Power control also improves the network throughput as summarized in Table III. Detailed results and figures are not presented due to space constraints.

\begin{tabular}{|c|c|c|}
\hline$\alpha$ & Throughput & Consumed Energy \\
\hline \hline 2 & $15.9 \%$ & $17.8 \%$ \\
\hline 3 & $29.2 \%$ & $34.48 \%$ \\
\hline
\end{tabular}

TABLE III: Throughput and Energy consumption gains

c) Throughput versus Energy Consumption: In figure 4, we show the energy consumption-throughput tradeoff for different values of $\mathrm{L}$, the number of power levels. For each value of $\alpha$, we show the corresponding energy consumed and throughput. The two extreme points $P_{0}\left(\mathcal{E}_{\text {min }}, \mathcal{R}_{\text {min }}\right)$ and $P_{1}\left(\mathcal{E}_{\max }, \mathcal{R}_{\max }\right)$ correspond to values of $\alpha=1$ which minimize the energy consumed and $\alpha=0$ which maximize the throughput, respectively. The curve between these two points represents the pareto-front, where each point of the front is derived for a given value of $\alpha$ as shown in the figure. Additionally, using power control improves the network performance.

In this section we provided simulation results of the optimization problem. We showed that the consumed energy and the throughput can be jointly optimized. Furthermore, we use the design parameter $\alpha$ to either focus on minimizing the consumed energy or on maximizing the throughput.

\section{CONCLUSION}

In this paper we have proposed a new joint energy consumption and throughput optimization model for directional antennas networks by considering a weighted objective function. 


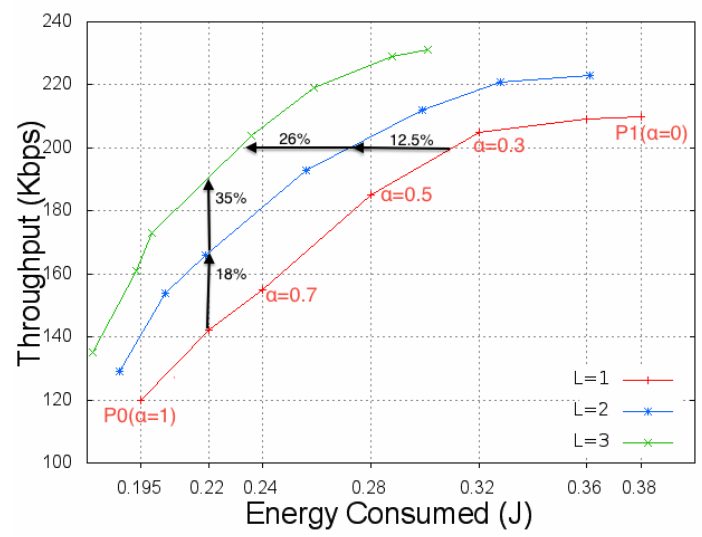

Fig. 4: Throughput vs Energy consumed for different values of $\mathrm{L}$

The considered optimization model takes into account different network constraints such as flow conservation, link capacity and loop avoidance constraints. We used ILOG Cplex solver to solve numerically the optimization problem. We showed that the energy consumption is an increasing function of the beamwidth. Furthermore, we showed that the more power levels we have, the less we consume energy. Additionally, we showed that the throughput-energy consumption tradeoff, for different values of $\alpha$, follows a Pareto front.

Proposing an heuristic based on Ant-Q optimization to reduce the complexity of the optimization problem is an interesting extension of this work.

\section{ACKNOWLEDGEMENT}

This work is part of the French project LCI4D of the Systematic Cluster, Paris Region Systems \& ICT Cluster (http://www.systematic-paris-region.org/).

\section{REFERENCES}

[1] I. I. CPLEX, "V12. 1: Users manual for cplex," International Business Machines Corporation, vol. 46, no. 53, p. 157, 2009.

[2] I. Akyildiz and X. Wang, "A survey on wireless mesh networks," Communications Magazine, IEEE, vol. 43, no. 9, pp. S23-S30, Sept 2005.

[3] I. Lahsen Cherif, L. Zitoune, and V. Veque, "Joint optimization of energy consumption and throughput of directional WMNs," in IEEE WiMob, New York City, New York, USA, 2016.

[4] A. Amokrane, R. Langar, R. Boutaba, and G. Pujolle, "Energy efficient management framework for multihop tdma-based wireless networks," Computer Networks, vol. 62, pp. 29 - 42, 2014. [Online]. Available: http://www.sciencedirect.com/science/article/pii/S138912861400005X

[5] A. Ouni, H. Rivano, F. Valois, and C. Rosenberg, "Energy and throughput optimization of wireless mesh networks with continuous power control," IEEE Transactions on Wireless Communications, vol. 14, no. 2 , pp. 1131-1142, Feb 2015.

[6] F. Moety, S. Lahoud, K. Khawam, and B. Cousin, "Joint power-delay minimization in green wireless access networks," in IEEE PIMRC, Sept 2013, pp. 2819-2824.

[7] A. H. Mohsenian-rad and V. W. S. Wong, "Joint logical topology design, interface assignment, channel allocation, and routing for multi-channel wireless mesh networks," IEEE Transactions on Wireless Communications, vol. 6, no. 12, pp. 4432-4440, December 2007.

[8] S. Boiardi, A. Capone, and B. Sansó, "Joint design and management of energy-aware mesh networks," Ad Hoc Networks, vol. 10, no. 7, pp. $1482-1496,2012$
[9] A. Capone, F. Malandra, and B. Sansò, "Energy savings in wireless mesh networks in a time-variable context," Mobile Networks and Applications, vol. 17 , no. 2 , pp. 298-311, 2012.

[10] W. Wong, X. Chen, F. Long, and S. H. G. Chan, "Joint topology control and routing assignment for wireless mesh with directional antennas," in Global Communications Conference (GLOBECOM), 2012 IEEE, Dec 2012, pp. 5699-5704.

[11] N. Sadeghianpour, T. C. Chuah, and S. W. Tan, "Joint channel assignment and routing in multiradio multichannel wireless mesh networks with directional antennas," International Journal of Communication Systems, vol. 28, no. 9, pp. 1521-1536, 2015. [Online]. Available: http://dx.doi.org/10.1002/dac.2731

[12] M. Żotkiewicz and M. Pióro, "Exact approach to reliability of wireless mesh networks with directional antennas," Telecommunication Systems, vol. 56, no. 1, pp. 201-211, 2013. [Online]. Available: http://dx.doi.org/10.1007/s11235-013-9829-4

[13] V. Ramamurthi, A. Reaz, S. Dixit, and B. Mukherjee, "Link scheduling and power control in wireless mesh networks with directional antennas," in Communications, 2008. ICC '08. IEEE International Conference on, May 2008, pp. 4835-4839.

[14] S. M. Das, H. Pucha, D. Koutsonikolas, Y. C. Hu, and D. Peroulis, "Dmesh: Incorporating practical directional antennas in multichannel wireless mesh networks," IEEE Journal on Selected Areas in Communications, vol. 24, no. 11, pp. 2028-2039, Nov 2006.

[15] B. Hu and H. Gharavi, "Directional routing protocols for ad-hoc networks," Communications, IET, vol. 2, no. 5, pp. 650-657, 2008.

[16] P. Gupta and P. Kumar, "The capacity of wireless networks," IEEE Transactions on Information Theory, vol. 46, no. 2, pp. 388-404, Mar 2000.

[17] B. Meindl and M. Templ, "Analysis of commercial and free and open source solvers for linear optimization problems," 2012.

\section{APPENDIX: VARIABLES/CONSTRAINTS LINEARIZATION}

In order to reduce the complexity of our problem and to use linear solvers for the optimization problem in (12), we proceed to variables linearization.

The energy consumption function in (9)

$$
\begin{aligned}
\mathcal{E}_{i, t} & =\sum_{l=1}^{\left|\mathcal{B}_{i}\right|} \sum_{L=1}^{|\mathcal{L}|} a_{i, t}^{l, L} \mathcal{E}_{\mathrm{TX}, L}+b_{i, t} \mathcal{E}_{\mathrm{RX}}+c_{i, t} \mathcal{E}_{\text {idle }}-b_{i, t} \cdot c_{i, t} \mathcal{E}_{\mathrm{idle}} \\
& -\sum_{l=1}^{\left|\mathcal{B}_{i}\right|} \sum_{L=1}^{|\mathcal{L}|} a_{i, t}^{m, L} \cdot c_{i, t} \mathcal{E}_{\text {idle }}+\sum_{l=1}^{\left|\mathcal{B}_{i}\right|} \sum_{L=1}^{|\mathcal{L}|} a_{i, t}^{m, L} \cdot c_{i, t} \cdot b_{i, t} \mathcal{E}_{\text {idle }} \\
+ & \sum_{l=1}^{\left|\mathcal{B}_{i}\right|} a_{i, t}^{l, 0} \mathcal{E}_{\text {idle }}
\end{aligned}
$$

For the term $b_{i, t} \times c_{i, t}$ can be linearized as follows:

$$
m_{i, t}=b_{i, t} \times c_{i, t} \quad \forall i \in \mathcal{I}, \quad \forall m \in \mathcal{B}_{i}
$$

where the binary variable (m) must satisfy the following constraints:

$$
\begin{array}{cc}
m_{i, t}-b_{i, t} \leq 0 & \forall i \in \mathcal{I}, \quad \forall m \in \mathcal{B}_{i} \\
m_{i, t}-c_{i, t} \leq 0 & \forall i \in \mathcal{I}, \quad \forall m \in \mathcal{B}_{i} \\
b_{i, t}+c_{i, t}-m_{i, t} \leq 1 & \forall i \in \mathcal{I}, \quad \forall m \in \mathcal{B}_{i}
\end{array}
$$

We follow the same process for $a_{i, t}^{m, L} \times c_{i, t}$ and $a_{i, t}^{m, L} \times$ $c_{i, t} \times b_{i, t}$ linearizations. 